\title{
Synthesis of phase-locked counter-phase modulated pumps for SBS-suppressed fiber parametric amplifiers
}

\author{
J. Kakande*, R. Slavík, F. Parmigiani, P. Petropoulos, and D. J. Richardson \\ Optoelectronics Research Centre, University of Southampton, Southampton, SO17 1BJ, UK \\ *jkk@orc.soton.ac.uk
}

\begin{abstract}
We propose and experimentally demonstrate a new all-optical technique for the generation of two optical pumps with oppositely varying carrier phases for mitigating SBS in parametric amplifiers without phase-dither transfer from pumps to signal. C2010 Optical Society of America

OCIS codes: (060.2320) Fiber optics amplifiers and oscillators, (190.4410) Nonlinear optics, parametric processes.
\end{abstract}

\section{Introduction}

The need to suppress stimulated Brillouin scattering (SBS) is currently a major limitation of fiber optic parametric amplifiers (FOPA) [1]. The most common approach to raise the SBS threshold is by dithering the pump phase thereby reducing the power spectral density. However, this is accompanied by detrimental spectral broadening of the idler wave, as well as gain distortions originating from the modulator rise and fall times [2-3]. Theoretically, the idler broadening can be fully suppressed by using a two-pump FOPA (2P FOPA) in which the two pumps are modulated using complementary phase patterns. This was demonstrated in various schemes directly employing a $\mathrm{LiNbO}_{3}$ modulator (with the optical delay between the two pumps precisely calibrated) [4] or two $\mathrm{LiNbO}_{3}$ modulators (driven by opposite electrical drive signals) [5]. These approaches are limited by the need for precise phase and amplitude synchronization of the modulating signals, as well as the finite device rise and fall times [6]. Recently, a customized dual wavelength push-pull style modulator [6] was demonstrated negating the need for the electrical signal synchronization in the previous schemes.

Phase sensitive amplifier (PSA) applications require pumps that are not only oppositely dithered but also have carriers locked in phase. In this paper we demonstrate a new all-optical configuration in which two counterphased pumps can be automatically phase locked to an incoming signal and can thus be directly used in a two pump FOPA without the need for dynamic pumps-signal phase synchronization. It is based on a cascaded process of second harmonic generation (SHG) followed by difference frequency generation (DFG) in a $30-\mathrm{mm}$ long periodically-poled $\mathrm{LiNbO}_{3}$ (PPLN) waveguide [7]. The use of the PPLN guarantees far greater immunity to the effects of SBS of the pump beams as well as the use of pumps with spectral separations of up to $80 \mathrm{~nm}$.

\section{Experimental Setup and Results}

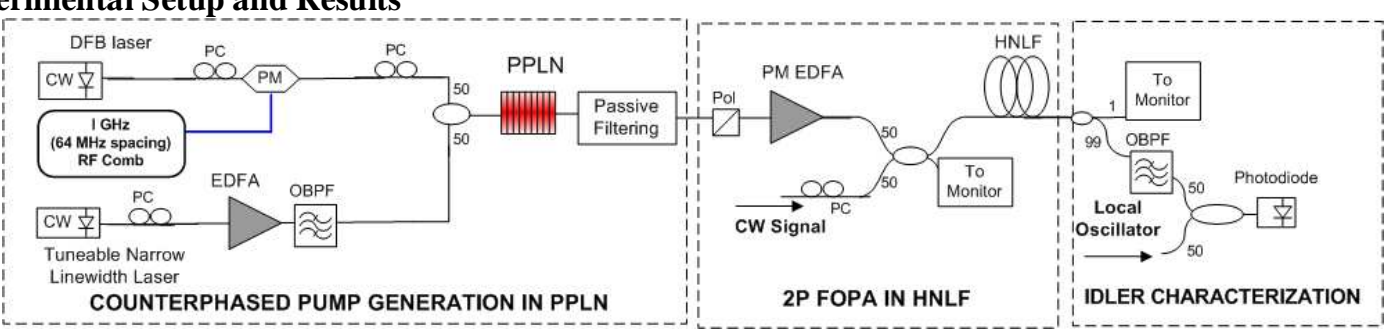

Figure 1: Phase sensitive amplifier setup, $\mathrm{CW}$ - continuous wave, $\mathrm{PM} \overline{\mathrm{EDFA}}-$ polarisation maintaining erbium doped fibre amplifier, PC - polarisation controller, PM - phase modulator, Pol - Polariser, OBPF - optical band pass filter.

A narrow linewidth ( 10 kHz) CW signal at $1539 \mathrm{~nm}$ (primary pump) was amplified to $20 \mathrm{dBm}$ and combined with another $\mathrm{CW}$ signal at $1545.5 \mathrm{~nm}$ (linewidth of $\sim 15 \mathrm{MHz}$ ), which was phase modulated using a $64 \mathrm{MHz}$ spaced electrical RF comb to broaden its linewidth to $1 \mathrm{GHz}$ (Secondary Pump). With this setup, the use of a $\mathrm{LiNbO}_{3}$ modulator could in the future be replaced by all-optical techniques such as cross phase modulation (XPM) in a nonlinear medium [8] to modulate the secondary pump. This would prevent the unwanted residual amplitude noise transfer to the modulated waves that is characteristic of $\mathrm{LiNbO}_{3}$ phase modulators, as this can significantly impair the noise performance of the 2P FOPA, more so in the PSA configuration.

Subsequently, the two waves were launched into the PPLN waveguide (coupling loss 1.4dB, propagation loss $0.3 \mathrm{~dB} / \mathrm{cm}$, nonlinear SHG coefficient $25 \mathrm{pm} / \mathrm{V}$ at $1064 \mathrm{~nm}$ ) with a total input power of $21 \mathrm{dBm}$ (limited by the device damage threshold). The phase matching wavelength of the PPLN device is $1545.5 \mathrm{~nm}$ at $44^{\circ} \mathrm{C}$. Due to the cascaded SHG/DFG process [7] an idler at $1552 \mathrm{~nm}$ is generated. If $\varphi_{p}, \varphi_{s}$ and $\varphi_{i}$ are the absolute phases of the 
primary pump, signal, and idler waves, respectively, then $\varphi_{i}=2 \varphi_{p}-\varphi_{s}$, implying that the idler and signal are counter-phased within the narrow limits imposed by the $2 \varphi_{p}$ term. In addition, if the primary pump is obtained from a recovered signal carrier then the $2 \varphi_{p}$ term indicates that the counter-phased pumps are phase locked to the signal, and could be directly used in a PSA. Due to the relatively low input powers, the generated idler power was $17 \mathrm{~dB}$ below that of the signal and therefore an intermediate stage, comprising an EDFA and passive filters, was required to reduce the signal-idler pair power difference followed by narrow filtering to remove the residual primary pump signal. The two remaining signals were the generated counter phased pumps that could be directly used in a subsequent FOPA, see Fig.2(a).

To evaluate the phase correlation of the counter-phased pumps and to show its usability in an FOPA, the following characterization was carried out. The pumps were amplified and coupled into a 500m HNLF (zero dispersion wavelength $1550 \mathrm{~nm}$, dispersion slope $0.03 \mathrm{ps} / \mathrm{nm}^{2} / \mathrm{km}$, nonlinearity 20/W/km and loss $0.53 \mathrm{~dB} / \mathrm{km}$ ). They were then combined with a $15-\mathrm{MHz}$ linewidth $1548 \mathrm{~nm} \mathrm{CW}$ signal to generate an idler at the wavelength of $1542 \mathrm{~nm}$ via the four wave mixing (FWM) process, see Fig. 2(b).
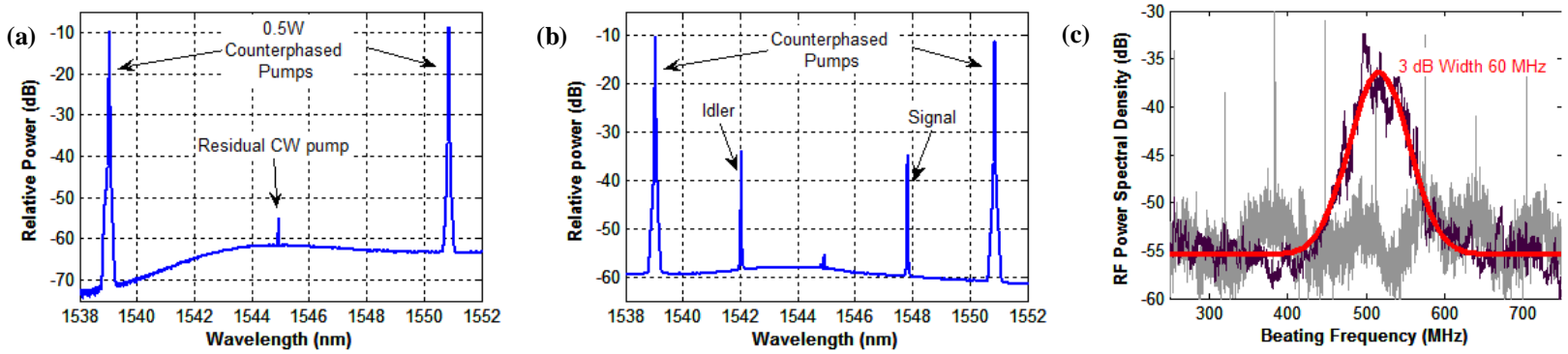

Figure 2(a) -- Counter phased pumps at the 2P FOPA HNLF input amplified in a PM EDFA with OSNRs exceeding 50 dB. 2(b) -- 2P FOPA output spectrum with idler OSNR over $25 \mathrm{~dB}$. 2(c) --Heterodyne signal generated by mixing idler and a local oscillator, thick solid line is 60 $\mathrm{MHz}$ fit, dashed grey line shows mixing result with both pumps co-phased in the same $\mathrm{LiNbO}_{3}$ modulator.

The idler, which had an optical signal-to-noise ratio (OSNR) better than $20 \mathrm{~dB}$, was then extracted at the output of the HNLF and subsequently heterodyned with a $\sim 15 \mathrm{MHz}$ linewidth $\mathrm{CW}$ laser of a slightly different carrier frequency (about $520 \mathrm{MHz}$ apart). The heterodyne signal is shown in Fig. 2(c) showing beating 3-dB width of 60 $\mathrm{MHz}$, which is within a factor of 2 of the anticipated minimum beating width. As a comparison, the dashed grey line in Fig. 2(c) shows the mixing result when two co-phased pumps are used in the same 2P FOPA (bypassing the PPLN), revealing the transfer of the pump dither including the strong $64 \mathrm{MHz}$ spaced tones to the idler and a linewidth exceeding the $750 \mathrm{MHz}$ measurement bandwidth. In addition, pump powers in excess of $30 \mathrm{dBm}$ were coupled into the 2P FOPA without exceeding the SBS threshold, indicating a threshold increase of at least $13 \mathrm{~dB}$.

\section{Conclusions}

We used a simple optical technique to generate counter-phased pumps for 2P FOPA applications with optical signalto-noise ratio $>50 \mathrm{~dB}$ and thereby drastically reduce the broadening experienced by the idler of a HNLF-based PSA. The use of parametric techniques to perform the counter-phasing allows for pumps that are automatically phase locked to an incoming signal.

This research has received funding from the European Communities Seventh Framework Programme FP/2007-2013 under grant agreement 224547 (PHASORS).

\section{References}

[1] J. Hansryd, et al., "Fiber-based optical parametric amplifiers and their applications," IEEE. J. Selected Topics Quantum Electron., vol. 8, no. 3, pp. 506-520,May/Jun. 2002.

[2] J. C. Boggio, et al.,"Q penalties due to pump phase modulation and pump RIN in fiber optic parametric amplifiers with non-uniform dispersion,” Opt.Commun., vol. 249, pp. 451-472, 2005.

[3] A. Durecu-Legrand, et al. "Impact of pump phase modulation on system performances of fiber optical parametric amplifiers,'IEE Electron. Lett., vol. 41, no. 6, pp. 83-84, Mar. 2005.

[4] S. Radic, et al., "Stimulated brillouin scattering suppression using a single modulator in two-pump parametric architectures,"presented at the Opt. Fiber Commun., Anaheim, CA, 2005.

[5] M. C. Ho, et al.,"Narrow-linewidth idler generation in fiber four-wave mixing and parametric amplification by dithering two pumps in opposition of phase," J. Lightw. Technol., vol. 20, no. 3, pp. 469-476, Mar. 2002.

[6] A. Vedadi, et al., "Demonstration of an Integrated LiNbO3 Synchronized Double Phase Modulator and Its Application to Dual-Pump Fiber Optical Parametric Amplifiers and Wavelength Converters," J. Lightwave Technol. 26, 777-781 (2008).

[7] K. Gallo, et al., "Efficient wavelength shifting over the erbium amplifier bandwidth via cascaded second order processes in lithium niobate waveguides," Appl. Phys. Lett. 71(8), 1020-1022 (1997).

[8] A. Mussot, et al., "All-optical SBS reduction in fiber optic parametric amplifiers”, Opt. Commun.,vol 282, Issue 5, pp 988-991, (2009). 